by repeated use of the operation, interspaced with other operations that act on single logical qubits only. Gao and colleagues realized the exponential-SWAP gate in an architecture composed of superconducting cavities that contain microwave fields and that are coupled and measured using superconducting electrical components called transmons.

In the authors' experiment, logical qubits are encoded in the states of multiple photons in two cavities - another infinite-dimensional system (Fig. 1b). Gao et al. realized two different encodings: Fock encoding, which is based on the number of photons; and coherent-state encoding, which uses the superposition (summation) of particular quantum states called coherent states. Notably, the latter encoding was previously shown to extend the lifetime of a logical qubit beyond what is achievable using any of its physical constituents alone ${ }^{9}$. On the basis of the experimental techniques that were developed to extend the lifetime, Gao and colleagues demonstrated the exponential-SWAP gate by implementing a controlled exchange of photons between the two cavities. Crucially, this operation is deterministic, and works with both Fock encoding and coherent-state encoding, reaching fidelities of $85 \%$ and $60 \%$, respectively.

These two experiments demonstrate two cornerstones of quantum computation using continuous variables: GKP-encoded qubits and the exponential-SWAP gate. Even if the current set-ups do not yet reach the quality required for a fully fledged error-correction scheme, they represent a major step towards this objective. In particular, improvements are required to increase the lifetime of the encoded qubits and to allow the possibility of concatenating many levels of error correction. A common trait of the experiments is that they use ancillary discrete-variable systems to manipulate the continuous variables. Hybrid continuous and discrete schemes can therefore, in principle, be devised to improve the performance of both experiments ${ }^{10}$.

Besides full error correction and large-scale computation, which are probably still some distance away, the techniques developed in these studies could have other applications. For example, GKP-encoded qubits can be used for high-resolution displacement sensing ${ }^{11}$, whereas exponential-SWAP gates integrated in a circuit might enable the demonstration of specific instances in which quantum computation outperforms its classical counterpart ${ }^{12}$. More generally, the exquisite level of control attained in these two very different architectures shows that infinite-dimensional systems, despite their analog character, are a cuttingedge contender in the race for advanced quantum information processing. Scientists now have a remarkable source of inspiration to see what could be achieved using other emerging continuous-variable architectures, such as optomechanical and electromechanical systems and integrated optics.
Alessandro Ferraro is at the School of Mathematics and Physics, Queen's University Belfast, Belfast BT7 1NN, UK.

e-mail:a.ferraro@qub.ac.uk

1. Flühmann, C. et al. Nature $566,513-517$ (2019).

2. Gao, Y. Y. et al. Nature 566, 509-512 (2019).

3. Nielsen, M. A. \& Chuang, I. L. Quantum Computation and Quantum Information: 10th Anniversary Edition (Cambridge Univ. Press, 2010).

4. Braunstein, S. L. \& van Loock, P. Rev. Mod. Phys. 77, 513-577 (2005)

5. Gottesman, D., Kitaev, A. \& Preskill, J. Phys. Rev. A
64, 012310 (2001).

6. Ferraro, A., Olivares, S. \& Paris, M. Gaussian States in Quantum Information (Bibliopolis, 2005).

7. Kienzler, D. et al. Science 347, 53-56 (2015).

8. Lau, H.-K. \& Plenio, M. B. Phys. Rev. Lett. 117, 100501 (2016).

9. Ofek, N. et al. Nature 536, 441-445 (2016)

10.Andersen, U. L., Neergaard-Nielsen, J. S., van Loock, P. \& Furusawa, A. Nature Phys. 11 713-719 (2015).

11.Duivenvoorden, K., Terhal, B. M. \& Weigand, D. Phys. Rev. A 95, 012305 (2017).

12.Aaronson, S. \& Arkhipov, A. Theory Comput. 9, 143-252 (2013).

\title{
CARDIOVASCULAR BIOLOGY
}

\section{Fresh twist in a biochemical whodunnit}

\begin{abstract}
Nine years ago, the compound kynurenine was reported to be responsible for the dilation of blood vessels during a potentially fatal inflammatory condition. New evidence has now identified the true culprit. SEE LETTER P.548
\end{abstract}

\section{DAVID A. KASS}

T $\mathrm{n}$ the life-threatening condition known as sepsis, the body responds to infection by inducing widespread biochemical changes that make the situation worse, some of which can lead to a severe decline in blood pressure. Several molecular factors that alter the constriction of blood vessels are involved in this decline, including nitric oxide, prostaglandins and oxidants such as hydrogen peroxide. In 2010, kynurenine - a metabolic product of the amino acid tryptophan - was identified ${ }^{1}$ as another factor that causes blood vessels to widen during sepsis. Stanley et al. ${ }^{2}$ (who work in the same laboratory as the researchers who identified kynurenine) now say on page 548 that they got the wrong culprit.

Stanley and colleagues' work begins as a classic whodunnit. The authors found that carefully purified kynurenine often did not cause blood-vessel widening (vasodilation) of isolated blood vessels in the context of inflammation, despite the previous report ${ }^{1}$. However, they consistently observed vasodilation using a mixture of tryptophan and either the enzyme indoleamine 2,3-dioxygenase 1 (IDO1) or singlet oxygen, a reactive oxygen species that is generated by IDO1. The expression of IDO1 is normally low in cell types other than immune cells, but can be upregulated by inflammatory proteins known as cytokines and by redox stress $^{3,4}$ - which means that IDO1 is often expressed in the presence of oxidants.

Both IDO1 and singlet oxygen are involved in the production of kynurenine from tryptophan (Fig. 1a), but also in the making of other metabolites. The authors' findings therefore suggested that another vasodilator was being formed. They hunted it down, and found it to be a compound that they call cis-WOOH (Fig. 1b), which is formed by IDO1 in a reaction involving tryptophan and singlet oxygen in the presence of hydrogen peroxide.

IDO1 activity is conventionally thought to be stimulated by chemical reducing agents and inhibited by hydrogen peroxide ${ }^{5}$. By contrast, Stanley et al. found that reducing agents did not generate $c i s-\mathrm{WOOH}$ in their system, whereas exposure to hydrogen peroxide did. In a series of clever chemistry experiments, the authors showed that: in the presence of hydrogen peroxide, IDO1 generates singlet oxygen, followed by cis-WOOH; the oxygen atoms that are added to tryptophan to form cis-WOOH are derived from singlet oxygen rather than hydrogen peroxide; and both IDO1 activity and singlet oxygen are required for tryptophan to elicit vasodilation.

In the previous work from the same group ${ }^{1}$, kynurenine was reported to dilate blood vessels by causing the signalling molecule cyclic guanosine monophosphate (cGMP) to activate an enzyme called protein kinase G1a (PKG1a, which has a dimeric structure assembled from two identical protein monomers). Stanley and co-workers observed that vasodilation mediated by cis-WOOH needs much less cGMP than does blood-vessel relaxation mediated by kynurenine, but still requires activation of PKG1a.

The authors determined that cis-WOOH activates PKG1a by oxidizing a specific cysteine amino-acid residue (Cys 42) in the enzyme. This causes a disulfide bond to form ${ }^{6}$ between the Cys42 residues in the monomers of PKG1a. This oxidation has also been reported to stimulate the enzyme's activity in a 
certain type of artery (resistance arteries) independently of cGMP levels ${ }^{6}$, and to contribute to the maintenance of normal blood pressure and to the ability of resistance arteries to dilate when exposed to hydrogen peroxide ${ }^{7}$.

The authors found that incubation of PKG1a with cisWOOH or with tryptophan in the presence of IDO1 induces the formation of the disulfide bond. Moreover, vasodilation of blood vessels isolated from a mouse model of sepsis - endotoxaemic mice, in which the animals are exposed to bacteria-derived toxins - was suppressed when the animals expressed a mutant version of PKG1a that could not dimerize at Cys 42 on exposure to oxidants. The authors then observed that upregulation and activation of IDO1 in a mouse model of atherosclerosis (an inflammatory disease that is characterized by narrowing of blood vessels) contributes to tryptophan-mediated vasodilation and blood-pressure control, which suggests that IDO1 could be a target in efforts to develop treatments for inflammatory diseases.

The discovery adds cisWOOH to a sizeable list of vasodilators that are involved in inflammatory responses, and reveals a specific role for singlet oxygen in the physiology of diseased mammalian cells (Fig. 1). Levels of IDO1 expression and activity must be high to form the singlet oxygen that, in turn, generates cis-WOOH; such conditions are common in inflamed tissues, but not healthy ones. The conversion of tryptophan to cis-WOOH therefore occurs under conditions of oxidative and inflammatory stress. Although hydrogen peroxide can cause vasodilation in the absence of IDO1 by inducing disulfide-bond formation between the Cys- 42 residues in PKG1a, the authors found that some of the relaxation of artery walls that was induced by hydrogen peroxide under normal conditions depends on the presence of IDO1.

The idea that kynurenine-induced vasodilation is caused by the activation of PKG1 a by $\mathrm{cGMP}^{1}$ now looks doubtful. Stanley et al. suggest that the vasodilation reported in the previous study was caused by contamination of kynurenine with cis-WOOH. However, the link between cis-WOOH and vasodilation does not depend on cGMP, but rather on PKG1 $\alpha$ oxidation, so the authors' proposal cannot explain the earlier findings.

Could selective inhibition of IDO1 be used therapeutically to reduce the large drop in blood pressure that is experienced by people who have sepsis? Perhaps, although several other approaches are also possible, given that many vasodilating factors are involved in systemic inflammation. Moreover, IDO1 has many crucial roles in immune cells, so its inhibition is likely to have several effects. For example, IDO1 inhibitors have been developed for use in cancer therapy ${ }^{8}-$ many tumours express IDO1, which helps them to evade the immune system by suppressing the functioning of certain immune cells and enhancing that of others ${ }^{9}$. Clinical trials of such compounds have been disappointing, in part because of the complexity of the pathway being targeted ${ }^{9}$. Nevertheless, it would be interesting to see whether those trials indicate that IDO1 inhibition can raise blood pressure, particularly in people who have inflammatory syndromes and elevated levels of kynurenine.

Another consideration with respect to the therapeutic use of IDO1 inhibitors is that IDO1 upregulation during sepsis might protect the body from a hyperactive immune system by increasing tolerance to endotoxaemia ${ }^{10}$. And although kynurenine and its metabolites are highly relevant to cancer and immune modulation $^{3,5,11}$, the extent to which tumours and the immune system are affected by tryptophan signalling through the pathway now identified by Stanley and co-workers remains to be seen.

Vasodilation by cis-WOOH ultimately derives from the oxidation of Cys 42 in PKG1a. However, Cys 42 might not be the only cysteine residue that can be oxidized to activate the enzyme; Cys 117 seems to be another ${ }^{12}$. The effects of the Cys 42 mutation that Stanley et al. used to prevent PKG1a dimerization are also controversial. Some studies $^{12,13}$ have found that it might depress cGMP-stimulated PKG1 $\alpha$ activity. Furthermore, PKG1 $\alpha$ dimerization triggered by Cys 42 oxidation occurs in the hearts of mice stressed by high blood pressure (pressure overload), yet its prevention in mice with the Cys 42 mutation enhances PKG1amediated protection against such stress $^{14}$ - not by altering PKG1 $\alpha$ activation, but by changing the intracellular localization of that enzyme and therefore its interactions with other proteins. Stanley and colleagues found that levels of IDO1 in heart-muscle tissue in mice were undetectable during pressure overload, but rose markedly in response to administration of interferon- $\gamma$ (a protein that is involved in many inflammatory responses), which suggests a role for IDO1-generated cis-WOOH in inflammatory conditions of the heart. These details will be interesting to unpick, as the plot of this vasodilation whodunnit thickens.

David A. Kass is in the Division of Cardiology of the Department of Medicine, the Department of Pharmacology and Molecular Sciences and the Department of Biomedical Engineering, The Johns Hopkins University School of Medicine, Baltimore, Maryland 21205, USA.

e-mail:dkass@jhmi.edu

1. Wang, Y. et al. Nature Med. 16, 279-285 (2010).

2. Stanley, C. P. et al. Nature 566, 548-552 (2019).

3. Baumgartner, R., Forteza, M. J. \& Ketelhuth, D. F. J. Cytokine http://dx.doi.org/10.1016/j. cyto.2017.09.004 (2017).

4. Changsirivathanathamrong, D. et al. Crit. Care Med. 39, 2678-2683 (2011).

5. Yeung, A. W., Terentis, A. C., King, N. J. \& Thomas, S. R. Clin. Sci. 129, 601-672 (2015).

6. Burgoyne, J. R. et al. Science 317, 1393-1397 (2007).

7. Prysyazhna, O., Rudyk, O. \& Eaton, P. Nature Med. 18, 286-290 (2012).

8. Komiya, T. \& Huang, C. H. Front. Oncol. 8, 423 (2018).

9. Zhai, L. et al. Cell. Mol. Immunol. 15, 447-457 (2018).

10.Wirthgen, E. \& Hoeflich, A. J. Amino Acids 2015 , 
973548 (2015).

11. Mangge, H. et al. Curr. Med. Chem. 21, 1931-1937 (2014).

12.Sheehe, J. L. et al. J. Biol. Chem. 293, 16791-16802 (2018).

13.Kalyanaraman, H., Zhuang, S., Pilz, R. B. \&
Casteel, D. E. J. Biol. Chem. 292, 8262-8268 (2017).

14. Nakamura, T. et al. J. Clin. Invest. 125, 2468-2472 (2015).

This article was published online on 13 February 2019.

\section{MATERIALS CHEMISTRY}

\section{Machines learn chemical intuition}

Failed chemical reactions are often not reported, which means that vast amounts of potentially useful data are going to waste. Experiments show that machine learning can use such data to optimize the preparation of porous materials.

\section{SETH COHEN}

T ntuition often guides our choices throughout life. In science, it can also play a part in the design of experiments to answer or probe a question of interest. For example, it guides chemists to select a specific set of reagents, reactions or conditions when devising the synthesis of a target compound. Writing in Nature Communications, Moosavi et al. ${ }^{1}$ report their use of machine learning to capture this sort of intuition to optimize the synthesis of an emerging class of material known as metal-organic frameworks (MOFs), which have applications as diverse as fuel storage, catalysis and the capture of water from the atmosphere.

To make a MOF, inorganic 'nodes' are connected by organic 'linker' molecules ${ }^{2}$. One can imagine these materials as molecular climbing frames, in which the linkers are the frames' metal rods and the nodes are the connections where the rods are riveted together. MOFs have large, extended molecular structures and consist mostly of empty space. The open voids in these structures make them among the most porous materials ever synthesized, and this underpins their many potential applications.

The wide variety of available linkers and nodes makes the number of MOFs that could be created nearly limitless - thousands have been synthesized in the past 20 years or so $^{3}$. However, for every reported synthesis of a MOF, dozens (and possibly even hundreds or thousands) of failed reactions will almost certainly have been attempted that did not produce the desired material. If this unreported wealth of knowledge of failed reactions could be captured, it could be used to predict and optimize the syntheses of new MOFs in the future.

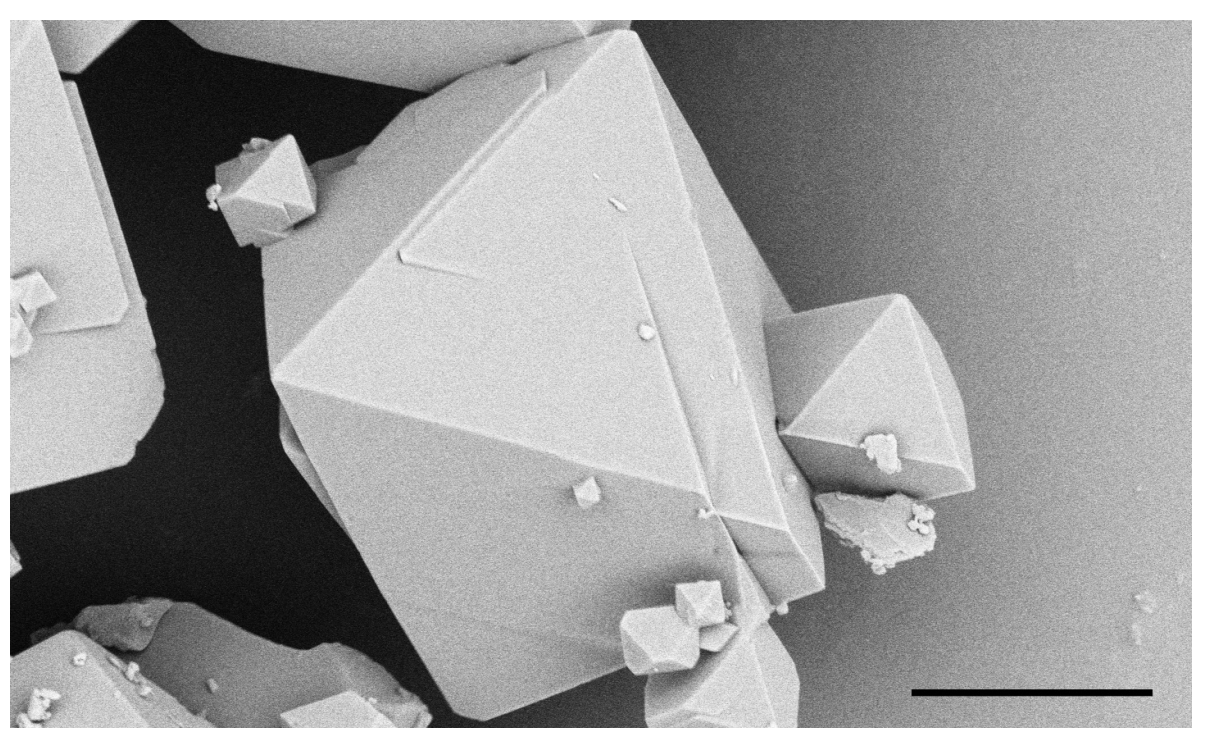

Figure 1 | Optimized synthesis of a metal-organic framework produces highly crystalline material. Moosavi et al. ${ }^{1}$ used machine learning to optimize the synthesis of HKUST-1, a porous material known as a metal-organic framework. This micrograph shows that the resulting material is highly crystalline, as required for potential applications. Scale bar, 5 micrometres. (Image from Supplementary Figure 11e of ref. 1.) 